\title{
Lifshitz Black Hole in Three Dimensions
}

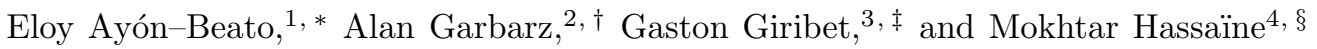 \\ ${ }^{1}$ Departamento de Física, CINVESTAV-IPN, Apdo. Postal 14-740, 07000, México D.F., México. \\ ${ }^{2}$ Departamento de Física, Universidad de Buenos Aires FCEN - UBA, \\ Ciudad Universitaria, Pabellón 1, 1428, Buenos Aires, Argentina. \\ ${ }^{3}$ Center for Cosmology and Particle Physics, at the Physics Department of \\ New York University CCPP - NYU, 4 Washington Place NY10003, USA. \\ ${ }^{4}$ Instituto de Matemática y Física, Universidad de Talca, Casilla 747, Talca, Chile.
}

\begin{abstract}
We show that three-dimensional massive gravity admits Lifshitz metrics with generic values of the dynamical exponent $z$ as exact solutions. At the point $z=3$, exact black hole solutions which are asymptotically Lifshitz arise. These spacetimes are three-dimensional analogues of those that were recently proposed as gravity duals for anisotropic scale invariant fixed points.
\end{abstract}

The enormous success of gauge-gravity duality [1] has triggered the interest in generalizing the holographic techniques to other areas of physics. Recently, the attempts to generalize AdS/CFT correspondence to nonrelativistic condensed matter physics have received considerable attention. Besides being an active line of research, this has given raise to very interesting new ideas; see Ref. [2] and references therein for a review.

Recently, candidates to be gravity duals for nonrelativistic scale invariant theories, both exhibiting Galilean invariance or not, have been proposed. In Refs. 3, 4], spacetimes whose isometry group is the so-called Schrödinger group were proposed to be gravity duals for Galilean and scale invariant systems. In Ref. [5], the scale invariant fixed points that do not exhibit Galilean symmetry were also analyzed, and the metric of the corresponding gravity duals were introduced (see Eq. (2) below). These metrics manifestly exhibit the anisotropic scale invariance

$$
t \mapsto \lambda^{z} t, \quad \vec{x} \mapsto \lambda \vec{x},
$$

which is characterized by the dynamical critical exponent $z$. The value $z=1$ corresponds to the standard scaling behavior of conformal invariant systems.

The typical example of a model with such a scaling symmetry is the Lifshitz model with $z=2$; this is the reason why the metrics of the proposed gravity duals are usually referred to as Lifshitz spacetimes. This type of Lifshitz fixed point appears in systems of strongly correlated electrons and other interesting problems in condensed matter physics. In turn, having a holographic description for these phenomena would be of great importance to describe condensed matter systems in the strongly coupled regime.

The basic idea is to look for spacetime geometries that incarnate the dynamical scaling symmetry (11). The

\footnotetext{
*Electronic address: ayon-beato-at-fis.cinvestav.mx

${ }^{\dagger}$ Electronic address: alan-at-df.uba.ar

‡Electronic address: gaston-at-df.uba.ar

$\S$ Electronic address: hassaine-at-inst-mat.utalca.cl
}

spacetimes that were identified as possible gravity duals for these Lifshitz fixed points are [5]

$$
d s^{2}=-\frac{r^{2 z}}{l^{2 z}} d t^{2}+\frac{l^{2}}{r^{2}} d r^{2}+\frac{r^{2}}{l^{2}} d \vec{x}^{2} .
$$

where $\vec{x}$ is a $d$-dimensional vector. It is simple to see that these spacetimes are invariant under the rescaling $(t, \vec{x}, r) \mapsto\left(\lambda^{z} t, \lambda \vec{x}, \lambda^{-1} r\right)$. It was also shown in [5] that metrics (2) with $d=2$ arise as solutions of General Relativity with a negative cosmological constant and $p$-form gauge fields as sources.

The proposal for the holographic prescription in [5] follows the standard AdS/CFT recipe. In the $(d+2)$ dimensional bulk defined by the spacetime (2), one considers a massive scalar mode whose asymptotic behavior in the near boundary limit takes the form $\varphi(r) \simeq$ $\varphi_{0}^{(-)} r^{-\Delta_{-}^{(z)}}+\varphi_{0}^{(+)} r^{-\Delta_{+}^{(z)}}$. This yields the relation between the mass of the scalar mode $m$ in the bulk and the conformal dimension $\Delta^{(z)}$ of the associated operator $\mathcal{O}_{\Delta^{(z)}}$ in the boundary theory, given by $\Delta_{ \pm}^{(z)}\left(\Delta_{ \pm}^{(z)}-z-d\right)=\mathrm{m}^{2} l^{2}$. As consequence, the Breitenlohner-Freedman type bound $\mathrm{m}^{2} l^{2}>-(z+d)^{2} / 4$ arises. Then, one considers the branch $\Delta_{-}^{(z)}$, whose fall-off does not spoil the asymptotic behavior of the metric at infinity. Finally, one is ready to match bulk and boundary observables computing correlators of physical operators. In [5], the case of a two-point correlation function $\left\langle\mathcal{O}_{\Delta^{(2)}}(x) \mathcal{O}_{\Delta^{(2)}}(y)\right\rangle$ was analyzed.

Of great importance is to introduce finite temperature effects in the story. With this motivation, black hole solutions in Lifshitz spaces (2) were also investigated in the literature. However, in spite of the apparently simple form of the spacetimes (2), the problem of finding analytic exact black hole solutions which asymptote these metrics turned out to be a highly non-trivial problem. In Ref. [6], a particular solution was found, which corresponds to a four-dimensional topological black hole which is asymptotically Lifshitz with dynamical exponent $z=2$. In Refs. [7, 8], numerical solutions were also explored. Lifshitz black holes were also studied in Refs. [9, 10, 11] , and while this letter was being finished the paper [12] just appeared, where a similar analytical solution was found for $z=2$ with $d=2$. The problem of embedding these black holes in string theory was 
addressed in Ref. [13], where a remarkable solution with $z=3 / 2$ was found. Moreover, from the analysis performed in Ref. 13], it becomes evident how difficult is generalizing the solution to other values of $z$. In particular, some no-go theorems for the string theory embedding have been discussed in Ref. [14].

The main result of this letter is to show the existence of black hole solutions of three-dimensional massive gravity that are asymptotically Lifshitz with $z=3$. In addition, we will also establish that the vacua of three-dimensional massive gravity includes metrics (2) for generic values of dynamical critical exponent $z$.

The theory we will consider is the so-called New Massive Gravity (NMG) [15], which has attracted much attention recently due to its very appealing properties. NMG is defined by supplementing Einstein-Hilbert action with the particular square-curvature terms which gives raise to field equations with a second order trace. At the linearized level, it is equivalent to the Fierz-Pauli action for a massive spin-2 particle in three dimensions, which turns out to be a unitary model. The space of solutions of the theory was studied recently, and it became clear that it includes geometries of great interest, like black holes, warped- $\mathrm{AdS}_{3}$ spaces, and AdS-waves.

The action of the NMG is [15]

$S=\frac{1}{16 \pi G} \int d^{3} x \sqrt{-g}\left[R-2 \lambda-\frac{1}{m^{2}}\left(R_{\mu \nu} R^{\mu \nu}-\frac{3}{8} R^{2}\right)\right]$.

The associated field equations read

$$
R_{\mu \nu}-\frac{1}{2} R g_{\mu \nu}+\lambda g_{\mu \nu}-\frac{1}{2 m^{2}} K_{\mu \nu}=0,
$$

where

$$
\begin{aligned}
K_{\mu \nu}= & 2 \square R_{\mu \nu}-\frac{1}{2} \nabla_{\mu} \nabla_{\nu} R-\frac{1}{2} \square R g_{\mu \nu}+4 R_{\mu \alpha \nu \beta} R^{\alpha \beta} \\
& -\frac{3}{2} R R_{\mu \nu}-R_{\alpha \beta} R^{\alpha \beta} g_{\mu \nu}+\frac{3}{8} R^{2} g_{\mu \nu}
\end{aligned}
$$

Here, we will consider $G=1 / 8$. It is also convenient to define the dimensionless parameters

$$
m^{2} l^{2}=y, \quad \lambda l^{2}=w .
$$

This gravity theory exhibits special properties at the points

$$
y= \pm 1 / 2
$$

In particular, at $y=+1 / 2$, the central charge associated to the Virasoro algebra that generates the group of asymptotic $\mathrm{AdS}_{3}$ symmetries vanishes. At this point, solutions with interesting properties have been exhibited in [16, 17] where the asymptotically $\mathrm{AdS}_{3}$ solutions were shown to present a relaxed fall-off at infinity [18, 19]. We will see below that $y=+1 / 2$ is precisely the point where the dynamical exponent $z$ of the solutions we find takes the value $z=1$. In this case, the Lifshitz spacetime becomes $\mathrm{AdS}_{3}$ as it can be seen also from the scaling property (1) which corresponds to that of the conformal group.

At $y=-1 / 2$, the theory also exhibits special properties. It was shown in [16] that at this point the scalar modes of gravitational waves in $\mathrm{AdS}_{3}$ space precisely saturates the Breitenlonher-Freedman bound (BF); this fact is closely related to the emergence of solutions with a relaxed fall-off, which are typically given by logarithmic asymptotic branches in convenient system of coordinates. Also for $y=-1 / 2$, the theory admits interesting black hole solutions 20, 21] that generalize the static BTZ black hole 22, 23]. These (former) black holes also present a weakened version of Brown-Henneaux $\mathrm{AdS}_{3}$ boundary conditions 24]. As we will show below, the dynamical exponent of our Lifshitz vacua when $y=-1 / 2$ corresponds to $z=3$, and this is precisely the point where we will also find exact analytic black hole solutions with Lifshitz asymptotic. For completeness, let us mention that NMG admits solutions with full or partial Schrödinger isometry [16]. These solutions correspond to three-dimensional analogues of the spacetimes considered in [3, 4] as gravity duals for cold atoms. The full Schrödinger group arises at the point $y=17 / 2$.

In this letter, we are concerned with spacetimes with no Galilean symmetry. We first analyze solutions that are of the Lifshitz-type [5]. It is possible to verify that the equations of motion (5) admit solutions of the form (2) for a generic dynamical exponent $z$. In fact, it parameterizes the mass and the cosmological constant as

$$
y=-\frac{1}{2}\left(z^{2}-3 z+1\right), \quad w=-\frac{1}{2}\left(z^{2}+z+1\right) .
$$

In other words, Lifshitz vacua exist for generic $z$ provided an appropriate tuning of the coupling constants. This implies that Lifshitz solutions with $z \neq 1$ are only possible if the coupling constants satisfy the conditions $\lambda l^{2} \leq-3 / 8$ and $m^{2} l^{2} \leq 5 / 8$. On the other hand, we also have the $\mathrm{AdS}_{3}$ vacua $z=1$, and its identifications, for a different relation between the coupling constants $w=$ $-[1+1 /(4 y)]$. Curvature invariants $R$ and $R_{\mu \nu} R^{\mu \nu}$ associated to solution (2) do depend on $z$; these are constant and yield the relation $g^{\mu \nu} K_{\mu \nu}=R_{\mu \nu} R^{\mu \nu}-\frac{3}{8} R^{2}=2 m^{2} \lambda$.

Let us turn to the problem of finding black hole solutions of NMG that are asymptotically Lifshitz. In order to accomplish this goal, we consider the following ansatz

$$
d s^{2}=-\frac{r^{2 z}}{l^{2 z}} F(r) d t^{2}+\frac{l^{2}}{r^{2}} H(r) d r^{2}+\frac{r^{2}}{l^{2}} d x^{2}
$$

where $F(r)$ and $H(r)$ are functions of the radial coordinate. We will demand these functions to obey $\lim _{r \rightarrow \infty} F(r)=\lim _{r \rightarrow \infty} H^{-1}(r)=1$ and to present a single-zero at a given radius $r=r_{+}$where the horizon would be located, namely $F\left(r_{+}\right)=H^{-1}\left(r_{+}\right)=0$.

Intriguingly, for $z=3$, which corresponds to the particular point $y=-1 / 2, w=-13 / 2$, the field equations with the appropriate asymptotic conditions turn out to 
be solved by

$$
F(r)=H^{-1}(r)=1-\frac{M l^{2}}{r^{2}},
$$

where $M$ is an integration constant. Then, the static asymptotically Lifshitz black hole for $z=3$ is given by

$$
d s^{2}=-\frac{r^{6}}{l^{6}}\left(1-\frac{M l^{2}}{r^{2}}\right) d t^{2}+\frac{d r^{2}}{\left(\frac{r^{2}}{l^{2}}-M\right)}+r^{2} d \phi^{2},
$$

where we have renamed $\phi=x / l$ and the identification $\phi=\phi+2 \pi$ have been considered. The metric (9) presents a curvature singularity at $r=0$ and a single event horizon located at

$$
r_{+}=l \sqrt{M}
$$

It is interesting to note that the $z=3$ Lifshitz scale symmetry, $t \mapsto \lambda^{3} t, x \mapsto \lambda x, r \mapsto \lambda^{-1} r$, is preserved provided the parameter is allowed to rescale as $M \mapsto$ $\lambda^{-2} M$.

Solution (9) presents curvature singularity at $r=0$. In fact, the curvature invariants diverge at the origin; namely

$$
R=-\frac{26}{l^{2}}+\frac{8 M}{r^{2}}, \quad R_{\mu \nu} R^{\mu \nu}=\frac{260}{l^{4}}-\frac{152 M}{l^{2} r^{2}}+\frac{24 M^{2}}{r^{4}} .
$$

The Hawking temperature associated to the black hole solution (9) can be easily computed by requiring regularity on the tip of the Euclidean geometry after periodic identification. This yields the result

$$
T_{H}=\frac{r_{+}^{3}}{2 \pi l^{4}}=\frac{M^{3 / 2}}{2 \pi l},
$$

which is consistent with the behavior $T_{H} \sim r_{+}^{z}$ found in other examples. A complete analysis of the thermodynamical properties would require a better understanding of the computation of conserved charges for these backgrounds in NMG and, in particular, of the counterterms involved. This will be discussed elsewhere [25].

It turns out that the radial configuration for a massive scalar field $\varphi(r)$ in the black hole background (9) can be explicitly found in terms of hypergeometric functions. Namely,

$$
\begin{aligned}
\varphi(r) & =\varphi_{0}^{(-)} F\left(k, 1+k ; 1+2 k ; \frac{M l^{2}}{r^{2}}\right) r^{-2-2 k} \\
& +\varphi_{0}^{(+)} F\left(-k, 1-k ; 1-2 k ; \frac{M l^{2}}{r^{2}}\right) r^{-2+2 k} .
\end{aligned}
$$

where $k=-\frac{1}{2} \sqrt{\mathrm{m}^{2} l^{2}+4}$ and $\mathrm{m}$ is the mass of the scalar field. Notice that this exhibits the expected behavior at large distances, which reproduces the falling of $\sim r^{-\Delta_{ \pm}^{(3)}}$ discussed above, as $\Delta_{ \pm}^{(3)}=2 \pm 2 k$. It is also worth noticing that the solution exhibits a logarithmic dependence at $k=0$, namely $\mathrm{m}^{2} l^{2}=-4$, where a BF-type bound is saturated. This is a common feature in similar solutions on AdS space.

The solution for the field configuration $\varphi(t, r, \phi)=$ $\Phi(r) e^{i k \phi-i \omega t}$, that also depends on $t$ and $\phi$, can be also explicitly found. In this case, the radial dependence $\Phi(r)$ can be expressed in terms of HeunC special functions, which are solutions to the Heun's Confluent differential equation.

Coefficients $\varphi_{0}^{( \pm)}$in the expression above are ultimately determined by imposing boundary conditions at the horizon; see the analysis of [12. Actually, analyzing the similarities with the case $z=2, d=2$ of [12 results interesting. As in 12], the scalar field equation in the background (9) admits an exact expression in terms of hypergeometric functions. The resemblance with the case $z=2, d=2$ would make possible to adapt the analysis of 12] to our example and arrive to similar conclusions, like the exclusion of ultralocal form for the dual correlators.

Of course, a black hole solution for the case $z=1$ is already known; it corresponds to the static BTZ black hole 22, 23.

$$
d s^{2}=-\frac{r^{2}}{l^{2}}\left(1-\frac{M l^{2}}{r^{2}}\right) d t^{2}+\frac{d r^{2}}{\left(\frac{r^{2}}{l^{2}}-M\right)}+r^{2} d \phi^{2} .
$$

In some sense, the solution (9) can be thought of as a cousin of the three-dimensional static BTZ black hole, which arises for a different value of the dynamical exponent $z$. Within the parametrization (7) the two black hole solutions $z=1$ and $z=3$ appear at the two special points (6) discussed above.

It is worth noticing that the black hole solution (9) is conformally equivalent to a black string solution of the form

$$
d s^{2}=\frac{r^{2}}{l^{2}}\left(-f(r) d t^{2}+\frac{d r^{2}}{f(r)}+d x^{2}\right)
$$

with $f(r)=\frac{r^{4}}{l^{4}}\left(1-\frac{M l^{2}}{r^{2}}\right)$, and where the coordinate $x=l \phi$ is again uncompactified by taking the universal covering of $\phi$. It can be seen that, by boosting this black string solution along the $x$ direction, one can construct a rotating version of the spacetime (9). This spinning version of the solution will be discussed somewhere else 25.

The fact of having found that the theory defined by action (3) admits Lifshitz solutions with generic values for the dynamical exponent $z$ is highly non-trivial. To illustrate how difficult finding such a solutions in a highercurvature theory can be, let us consider the case of fivedimensional Einstein-Gauss-Bonnet theory

$$
\begin{aligned}
S= & \frac{1}{2 \pi} \int d^{5} x \sqrt{-g}\left[R+12 l^{-2}-\xi l^{2}\left(R^{2}-4 R_{\mu \nu} R^{\mu \nu}+\right.\right. \\
& \left.\left.R_{\mu \nu \alpha \beta} R^{\mu \nu \alpha \beta}\right)\right],
\end{aligned}
$$

which is a simpler example as it yields field equations of second order. It can be shown that, for generic values of 
the coupling constant $\xi$, this theory only admits solutions of the Lifshitz type for $z=1$; that is, the only solution corresponds to locally $\mathrm{AdS}_{5} \cdot{ }^{1}$ The only point where curious features arise is for $\xi=1 / 4$, where the theory suffers enhancement of symmetry. Indeed, for $\xi=1 / 4$, the action (14) can be written as a five-dimensional ChernSimons action, and thus it enjoys local gauge invariance under $S O(2,4)$ group. At this point, Lifshitz solutions are admitted for generic values of $z$ due to the wellknown degeneracies of the Chern-Simons theory [26]; see also 27] for a consideration of the $I S O(1,4)$ invariant Chern-Simons theory $1 / \xi=0$. This degeneracy in the value of the dynamical exponent is closely related to the non-renormalization of $z$ that happens at $\xi=1 / 4$, which was implicitly observed in [28]. Nevertheless, even at this special point where degeneracy in $z$ arises, asymptotically Lifshitz black holes do not seem to exist (for $z \neq 1$ ). This makes the solution (9) of particular interest.

Besides its uses as a remarkably simple model to explore the $d>1$ analogues, it could be interesting to investigate whether a more direct application of this $d=1$ Lifshitz solution to condensed matter physics exists. In particular, exploring the relation to the models discussed in 29] would be interesting.

Finally, one might also wonder whether a wider sector of Lifshitz fixed points arises if one considers the

${ }^{1}$ G.G. is grateful to Matías Aiello for a discussion about this point. generalization of three-dimensional massive gravity that amounts to including the Cotton tensor in the gravity action [15, 30]. After all, one knows that, in certain cases, the inclusion of the Cotton tensor produces the enlargement of the space of allowed configurations [16]. However, one can verify that this is not the case of the Lifshitz solutions. Remarkably, the addition of the Cotton tensor in the action generically excludes the Lifshitz configurations, as the only cases that are allowed correspond to $z=0$ and $z=1$.

\section{Acknowledgments}

The authors thank M. Aiello, J. Oliva and R. Troncoso for conversations. The work of A.G. is supported by University of Buenos Aires. G.G. is Member of the CICCONICET, Argentina, on leave of absence from University of Buenos Aires. This work has been partially supported by grant 1090368 from FONDECYT, by the project Redes de Anillos R04 from CONICYT, by grant UBACyT X861 from UBA, by grant PICT 00849 from ANPCyT, and by grants 82443 and $45946-\mathrm{F}$ from CONACyT.
[1] J. Maldacena, The Large $N$ Limit of Superconformal Field Theories and Supergravity, Adv. Theor. Math. Phys. 2 (1998) 253, arXiv:hep-th/9711200.

[2] S. Hartnoll, Lectures on holographic methods for condensed matter physics, arXiv:0903.3246.

[3] D.T. Son, Toward an AdS/cold atoms correspondence: a geometric realization of the Schroedinger symmetry, Phys. Rev. D78 (2008) 046003, arXiv:0804.3972.

[4] K. Balasubramanian and J. McGreevy, Gravity duals for non-relativistic CFTs, Phys. Rev. Lett. 101 (2008) 061601, arXiv:0804.4053.

[5] S. Kachru, X. Liu and M. Mulligan, Gravity Duals of Lifshitz-like Fixed Points, Phys. Rev. D78 (2008) 106005, arXiv:0808.1725.

[6] R. Mann, Lifshitz Topological Black Holes, arXiv:0905.1136.

[7] G. Bertoldi, B. Burrington and A. Peet, Black Holes in asymptotically Lifshitz spacetimes with arbitrary critical exponent, arXiv:0905.3183.

[8] G. Bertoldi, B. Burrington and A. Peet, Thermodynamics of black branes in asymptotically Lifshitz spacetimes, arXiv:0907.4755.

[9] M. Taylor, Non-relativistic holography, arXiv:0812.0530.

[10] U. Danielsson and L. Thorlacius, Black holes in asymptotically Lifshitz spacetime, JHEP 0903 (2009) 070, arXiv:0812.5088.

[11] E. Brynjolfsson, U. Danielsson, L. Thorlacius and T. Zingg, Holographic Superconductors with Lifshitz Scaling, arXiv:0908.2611.
[12] K. Balasubramanian and J. McGreevy, An analytic Lifshitz black hole, arXiv:0909.0263.

[13] T. Azeyanagi, W. Li and T. Takayanagi, On String Theory Duals of Lifshitz-like Fixed Points, arXiv:0905.0688.

[14] W. Li, T. Nishioka and T. Takayanagi, Some No-go Theorems for String Duals of Non-relativistic Lifshitz-like Theories, arXiv:0908.0363.

[15] E. Bergshoeff, O. Hohm and P. Townsend, Massive Gravity in Three Dimensions, Phys. Rev. Lett. 102 (2009) 201301, arXiv:0901.1766.

[16] E. Ayón-Beato, G. Giribet and M. Hassaïne, Bending AdS Waves with New Massive Gravity, JHEP 0905 (2009) 029, arXiv:0904.0668.

[17] G. Clément, Black holes with a null Killing vector in three-dimensional massive gravity, arXiv:0905.0553.

[18] Y. Liu and Y-W Sun, Note on New Massive Gravity, JHEP 0904 (2009) 106, arXiv:0903.0536.

[19] Y. Liu and Y-W Sun, Consistent Boundary Conditions for New Massive Gravity in $A d S_{3}$, arXiv:0903.2933.

[20] E. Bergshoeff, O. Hohm and P. Townsend, More on Massive 3D Gravity, Phys. Rev. D79 (2009) 124042, arXiv:0905.1259.

[21] J. Oliva, D. Tempo and R. Troncoso, Three-dimensional black holes, gravitational solitons, kinks and wormholes for BHT massive gravity, JHEP 0907 (2009) 011, arXiv:0905.1545.

[22] M. Banãdos, C. Teitelboim and J. Zanelli, The Black Hole in Three Dimensional Space Time, Phys. Rev. Lett. 69 (1992) 1849, arXiv:hep-th/9204099.

[23] M. Banãdos, M. Henneaux, C. Teitelboim and J. Zanelli, 
Geometry of the 2+1 Black Hole, Phys. Rev. D48 (1993) 1506, arXiv:gr-qc/9302012.

[24] J.D. Brown and M. Henneaux, Central Charges in the Canonical Realization of Asymptotic Symmetries: An Example from Three-Dimensional Gravity, Commun. Math. Phys. 104, 207 (1986).

[25] E. Ayón-Beato, A. Garbarz, G. Giribet and M. Hassaïne, in preparation.

[26] G. Dotti, J. Oliva and R. Troncoso, Static wormhole solution for higher-dimensional gravity in vacuum, Phys. Rev. D75 (2007) 024002, arXiv:hep-th/0607062.
[27] D-W Pang, $R^{2}$ Corrections to Asymptotically Lifshitz Spacetimes, arXiv:0908.1272.

[28] A. Adams, A. Maloney, A. Sinha and S. Vásquez, 1/N Effects in Non-Relativistic Gauge-Gravity Duality, JHEP 0903 (2009) 097, arXiv:0812.0166.

[29] Yu Nakayama, Gravity Dual for Reggeon Field Theory and Non-linear Quantum Finance, arXiv:0906.4112.

[30] S. Deser, R. Jackiw and S. Templeton, ThreeDimensional Massive Gauge Theories, Phys. Rev. Lett. 48 (1982) 975. 